\title{
Real World Evidence of Safety and Effectiveness of Combination of Vitamin E and Fraxinus excelsior in Treatment of Indian Patients with Nonalcoholic Fatty Liver Disease
}

\author{
Virukalpatti Gopalratnam Mohan Prasad1, Prashant Rahate ${ }^{2}$, Husain Bohri ${ }^{3}$, \\ Jyoti Ranjan Mahapatra4, Ashish Mungantiwar ${ }^{5}$, Priyanka Srivastava ${ }^{5}$, \\ Nirali Bhatt5 ${ }^{5}$, Dhavalkumar Patel ${ }^{5}$, Soumen Roy ${ }^{6 *}$, Amit Qamra ${ }^{6}$ \\ ${ }^{1}$ VGM Gastro Centre, Singanallur, Coimbatore, Tamil Nadu, India \\ ${ }^{2}$ SevenStar Hospital, Nagpur, Maharashtra, India \\ ${ }^{3}$ Premium Digestive Diesase Liver and Endoscopy Clinic, Nashik, Maharashtra, India \\ ${ }^{4}$ Apollo Clinic, Salt Lake City, Kolkata, West Bengal, India \\ ${ }^{5} \mathrm{R} \&$ D and Clinical Trials Department, Macleods Pharmaceuticals Ltd., Mumbai, Maharashtra, India \\ ${ }^{6}$ Medical Affairs Department, Macleods Pharmaceuticals Ltd., Mumbai, Maharashtra, India \\ Email: ^drsoumenr@macleodspharma.com
}

How to cite this paper: Prasad, V.G.M., Rahate, P., Bohri, H., Mahapatra, J.R., Mungantiwar, A., Srivastava, P., Bhatt, N., Patel, D., Roy, S. and Qamra, A. (2020) Real World Evidence of Safety and Effectiveness of Combination of Vitamin $\mathrm{E}$ and Fraxinus excelsior in Treatment of Indian Patients with Nonalcoholic Fatty Liver Disease. Open Journal of Gastroenterology, 10, 14-22. https://doi.org/10.4236/ojgas.2020.101002

Received: December 7, 2019

Accepted: January 5, 2020

Published: January 8, 2020

Copyright ( 2020 by author(s) and Scientific Research Publishing Inc. This work is licensed under the Creative Commons Attribution International License (CC BY 4.0).

http://creativecommons.org/licenses/by/4.0/ (c) (i) Open Access

\begin{abstract}
Introduction: Nonalcoholic fatty liver disease (NAFLD) is a chronic liver disease ranging from liver steatosis to nonalcoholic steatohepatitis (NASH). Besides lifestyle modifications, Vitamin E (800 IU/day) is generally recommended for NASH. Vitamin E monotherapy is not sufficient for the multifaceted disease like NALFD. The combination of Vitamin E 400IU and Fraxinus excelsior $500 \mathrm{mg}$ twice daily was found to be better than vitamin E $400 \mathrm{IU}$ twice daily in improving the lipid profile and liver function parameters in patients with NAFLD. We conducted a study to assess safety and effectiveness of Vitamin E plus Fraxinus excelsior in Indian patients with NAFLD in real-world settings. Patients and Methods: This was a non-interventional study in NAFLD patients with varying grades of steatosis conducted by 234 physicians across India from January 2018 to August 2018. Patients received combination of Vitamin E (400 IU) and Fraxinus excelsior (500 mg) soft gelatin capsules twice daily after meals for 12 weeks. Effectiveness of the treatment was assessed at visit 2 (6 weeks) and visit 3 (12 weeks, end of study) from baseline. The parameters for assessment included severity of liver steatosis, liver function parameters and the global assessment of safety and effectiveness. Results: A total of 1114 patients were included in the study. At baseline, majority of the patients $(71.18 \%)$ had Grade II liver steatosis followed by $21.01 \%$ and $7.81 \%$ patients who had
\end{abstract}


Grade III and Grade I liver steatosis, respectively. After 12 weeks of treatment with vitamin $\mathrm{E}$ and Fraxinus excelsior combination, $21 \%$ patients had no steatosis, $58.79 \%$ patients were in Grade 1 steatosis, $19.57 \%$ in grade II steatosis and only $0.63 \%$ patients in Grade III steatosis. The mean percentage reduction in aspartate aminotransferase (AST) level at week 6 and week 12 from baseline was $24.92 \%$ and $43.79 \%$, respectively. Similarly, the mean percentage reduction in alanine aminotransferase (ALT) level at week 6 and week 12 from baseline was $24.37 \%$ and $44.05 \%$ respectively. The mean reductions in AST and ALT were significant at week 6 and week 12. More than $50 \%$ of the investigators rated treatment as excellent for the safety and effectiveness. Conclusion: Evidence from this Indian real-life study suggests that Vitamin E (400 IU) and Fraxinus excelsior $(500 \mathrm{mg}$ ) is safe and effective for the treatment of NAFLD in routine clinical practice. Its consumption is associated with improvement in hepatic steatosis and liver function parameters (AST and ALT). Given the limited therapeutic options in NAFLD, this combination has the potential to bridge the therapeutic gap in management of NAFLD.

\section{Keywords}

NAFLD, NASH, Vitamin E, Fraxinus excelsior, Real Life, India

\section{Introduction}

Nonalcoholic fatty liver disease (NAFLD) is one of the major causes of chronic liver disease worldwide, with prevalence ranging from $6.3 \%$ - 33\% in general population [1]. NAFLD refers to abnormal accumulation of triglycerides in the liver (liver steatosis) due to causes other than excessive alcohol consumption. The spectrum of the disease ranges from simple steatosis (fatty liver) to nonalcoholic steatohepatitis (NASH) which may further progress to cirrhosis and hepatocellular carcinoma [1] [2].

NAFLD prevalence in Asian countries ranges from 12\% - 24\% and is associated with age, gender, locality and ethnicity [2] [3]. In India, the prevalence is up to $32 \%$ in general population and higher incidence is reported in obese (57.5\% - 74\%) and diabetic (56.5\%) populations [4].

Inspite of the high prevalence of NAFLD in Asian countries and globally, there is currently no definitive treatment for NAFLD. Besides lifestyle modifications, physical exercise and dietary control, there are no US Food and Drug Administration-approved medications for patients with NAFLD; progressing to non-alcoholic steatohepatitis (NASH) [1].

A randomized controlled trial (PIVENS trial) was reported by Sanyal AJ et al. in 2010 which proved the benefits of vitamin E (800 IU/day) in NAFLD patients progressing to NASH [5]. The common European ash biologically known as Fraxinus excelsior (F. excelsior), is a native to most of Europe and found in southwest Asia. Evidence suggests that extracts of $F$. excelsior promote insulin sensitivity and increase adiponectin-leptin ratio thereby reducing fat mass and body 
weight. F. excelsior has also exhibited beneficial effects in improving dyslipidemia [6].

The progression of the disease from simple steatosis to cirrhosis and further liver cancer is governed by "Two-Hit Hypothesis". Vitamin E monotherapy is not sufficient for multifaceted diseases like NAFLD. Vitamin E, being an antioxidant, targets NASH (Hit 2 stage) [7] [8]. There are no FDA approved medications currently which work on both steatosis (Hit 1 stage) and NASH (Hit 2 stage) in treatment of NAFLD [1]. Since F. excelsior and Vitamin E work on Hit 1/steatosis stage (Triglyceride accumulation/Insulin resistance) and Hit 2/Nonalcoholic Steatohepatitis (oxidative stress and inflammation) respectively, the fixed dose combination of Vitamin E (400 IU) and F excelsior (500 mg) soft gelatin capsule (Ensules ${ }^{+}$, Macleods Pharmaceuticals Limited, Mumbai, India) was evaluated for safety and efficacy compared to Vitamin E monotherapy in patients with NAFLD by Patil et al. [6]. The study reported that the fixed dose combination of Vitamin E (400 IU) and F. excelsior (500 mg) was more effective than vitamin $\mathrm{E}$ monotherapy in improving the lipid profile and liver function parameters in patients with NAFLD [6].

Real life studies reflect how treatments/interventions are administered in routine clinical practice and inform on the "effectiveness" of a treatment which is a measure of the extent to which an intervention does what is intended to do in routine real world clinical scenario. An additional advantage of real life studies is their natural clinical practice setting, e.g., physicians' office/Clinics, which ensures that they have external validity. Involvement of patients from different settings increases the variability of the results, but also reproduces the complexity of the health care system more reliably than the controlled conditions in Randomised Clinical trials (RCTs) [9].

Here we report a non-interventional, prospective, multicentric, Indian real life study to assess safety and effectiveness of a fixed dose combination of Vitamin $\mathrm{E}$ (400 IU) and F. excelsior (500 mg) soft gelatin capsule (Ensules ${ }^{+}$, Macleods Pharmaceuticals Limited, Mumbai, India) in patients with NAFLD.

\section{Patients and Methods}

The aim of the present non-interventional study was to assess the safety and effectiveness of Ensules ${ }^{+}$in Indian patients with NAFLD in a "real-life" scenario. Global safety and effectiveness, as judged by the investigator, were also evaluated at the end of the study.

\section{Study design}

This was an Indian multicentric, non-interventional, real-life study conducted by 234 Medical practitioners across India. The study was conducted from January 2018 to August 2018.

\section{Selection criterion}

Being a non-interventional, real-life study all patients of either sex who were diagnosed with NAFLD using clinical, bio-chemical and radiological (ultrasonography) parameters and were prescribed Ensules ${ }^{+}$soft gelatin capsules as part 
of routine clinical practice and care were enrolled in the study. There were no other inclusion/exclusion criterion.

\section{Study population}

Total of 1114 patients who were diagnosed with NAFLD using clinical, biochemical and ultrasonography parameters and prescribed Ensules ${ }^{+}$soft gelatin capsules by investigating physicians as per routine clinical practice, were included in the analysis.

\section{Treatment}

Patients were treated with Ensules ${ }^{+}$soft gelatin capsules two times a day after meals for 12 weeks as per routine clinical practice. General care and concomitant medications were allowed as per routine clinical practice and care.

\section{Study visits}

All patients were assessed at baseline (Visit 1), week 6 (Visit 2) and week 12 (Visit 3) for physical examination and laboratory bio-chemical evaluation (Liver Function tests-AST and ALT) as per routine clinical practice. Ultrasonographic evaluation of liver and spleen were performed on Visit 1 and Visit 3 by a radiologist for grading of steatosis. The global safety and effectiveness by the investigator were evaluated at the end of the study.

\section{Sample Size and Statistical Analysis:}

There was no formal sample size calculation. The continuous data is presented as mean and standard deviation; whereas, categorical data is presented as frequency and percentages. Comparisons with baseline was done using paired " $t$ " test.

\section{Results}

A total of 1114 patients with NAFLD were enrolled in the study. The demographics are shown in Table 1. The male to female ratio was 4.3:1 indicating a male majority with NAFLD reflecting sedentary lifestyle. The mean age was 48.17 years indicating a middle age population. The mean weight was $73.47 \mathrm{kgs}$, indicating an above average weight by Indian standards as expected in NAFLD population. Patients' baseline demographic data along with grades of steatosis and laboratory parameters were evaluated for the safety and effectiveness. Patients received Ensules ${ }^{+}$soft gelatin capsule twice daily after meals for 12 weeks.

Table 1. Demographic and baseline characteristics.

\begin{tabular}{cc}
\hline \multicolumn{2}{c}{ Demographic and Baseline Characteristics } \\
\hline No. of patients & 1114 \\
Gender* & \\
Male & 878 \\
Female & 201 \\
Mean age (years) & 48.17 \\
Mean weight (kgs) & 73.47 \\
\hline
\end{tabular}

${ }^{*}$ Data for 35 patients was not available. 


\section{Effectiveness Assessments:}

\section{Liver steatosis}

A total of 1114 patients' data was available for evaluation of severity of liver steatosis at baseline and two follow-up visits. At baseline, 7.81\%, 71.18\% and $21.01 \%$ patients had grade I, grade II or grade III of liver steatosis respectively. There was a significant reduction $(\mathrm{p}<0.05)$ in the number of patients with grade II and grade III after 6 week and 12 week treatment with Ensules ${ }^{+}$soft gelatin capsules. Briefly, the grade II patients were reduced to $49.64 \%$ and $19.57 \%$ at week 6 and week 12 respectively from baseline. Similarly, grade III patients were reduced to $8.62 \%$ and $0.63 \%$ at week 6 and week 12 respectively. In contrast, the patients in grade I increased to $41.74 \%$ and $58.79 \%$ at week 6 and week 12 from baseline (Figure 1) respectively. At the end of 12 weeks of treatment, no signs of liver steatosis were reported in $21 \%$ patients (Table 2 ).

At the end of 12 weeks, overall, 58.79\% patients were in Grade 1 steatosis, $19.57 \%$ were in Grade II steatosis, with only $0.63 \%$ patients in Grade III steatosis. By the end of study, $21 \%$ patients had absence of steatosis. These findings are clinically relevant as majorly the patients were in Grade I steatosis (58.79\%), followed by no steatosis (21\%) (Figure 1) by the end of study (12 weeks).

\section{Liver function parameters}

The effect of Ensules ${ }^{+}$on liver function parameters (AST and ALT) in NAFLD patients were assessed on Week 6 and Week 12 for 974 evaluable patients. The mean levels of AST and ALT at baseline were 75.71 and $76.85 \mathrm{IU} / \mathrm{L}$, respectively. Upon treatment with Ensules ${ }^{+}$soft gelatin capsules, the elevated levels of AST and ALT in NAFLD patients significantly reduced ( $\mathrm{p}<0.05$ ) to 56.84 and $58.12 \mathrm{IU} / \mathrm{L}$, respectively at week 6 and 42.56 and 42.99, respectively at week 12 (Table 3).

The mean reduction in AST following week 6 and week 12 treatment was $24.92 \%$ and $43.79 \%$ respectively. Similarly, the mean reduction in ALT following week 6 and week 12 treatment was $24.37 \%$ and $44.05 \%$ respectively (Figure 2).

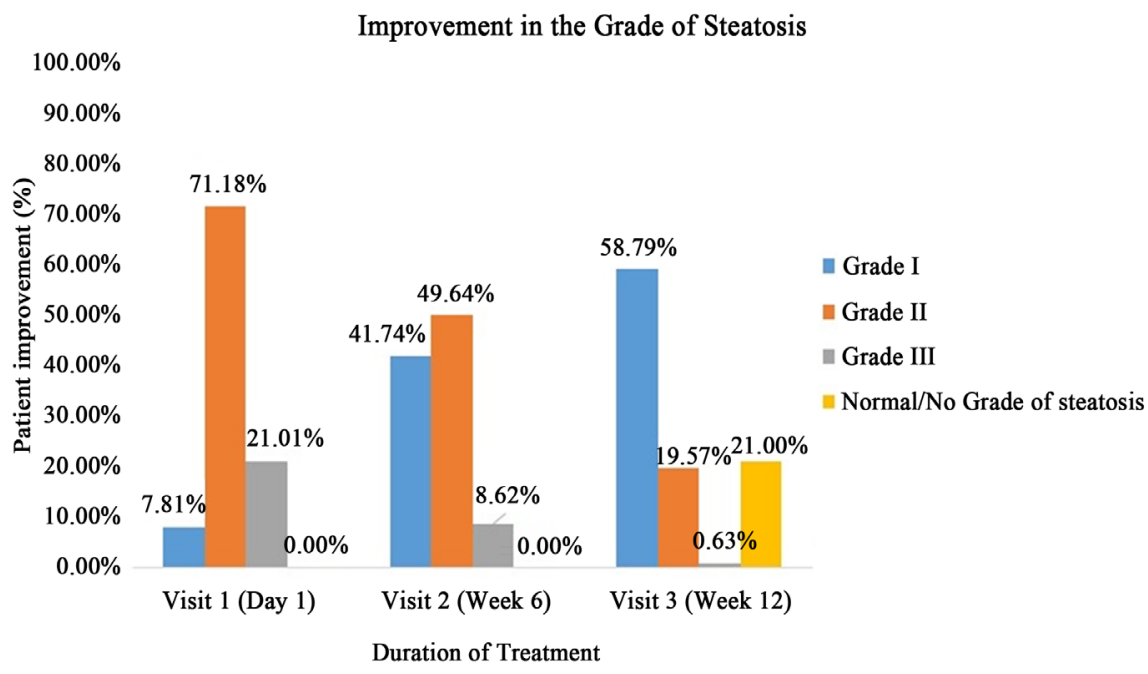

Figure 1. Improvement in liver steatosis from Visit 1 to Visit 3. 
Mean Reduction in AST and ALT levels

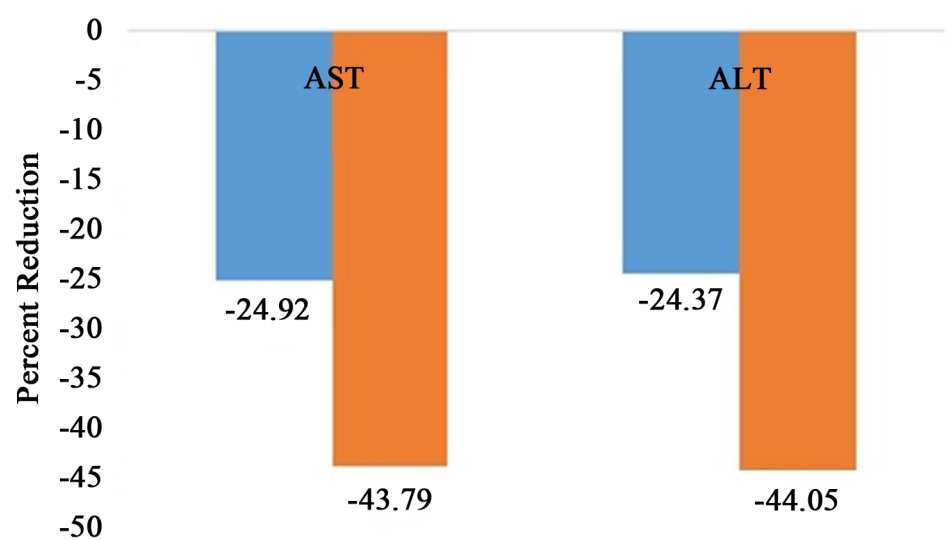

Week 6

Week 12

Figure 2. Percentage reductions in mean AST and ALT levels following Ensules ${ }^{+}$treatment.

Table 2. Percentage change in number of patients in severity of liver steatosis from baseline (Visit 1) to Visit 2 and Visit 3.

\begin{tabular}{ccccc}
\hline Grade of Liver Steatosis (n= 1114) & Grade I & Grade II & Grade III & $\begin{array}{c}\text { Normal/No. } \\
\text { Grade of Steatosis }\end{array}$ \\
\hline Visit 1 (Day 1) & $7.81 \%$ & $71.18 \%$ & $21.01 \%$ & 0 \\
Visit 2 (Week 6 \pm 2 days) & $41.74 \%$ & $49.64 \%$ & $8.62 \%$ & 0 \\
Visit 3 (Week 12 \pm 2 days) & $58.79 \%$ & $19.57 \%$ & $0.63 \%$ & $21.00 \%$ \\
\hline
\end{tabular}

Table 3. Effect of vitamin E (400 IU) and F excelsior $(500 \mathrm{mg})$ soft gelatin capsule capsules on ALT and AST levels of NAFLD patients.

\begin{tabular}{cccc}
\hline Liver Function Parameter $(\mathrm{n}=974)$ & Baseline & Week 6 & Week 12 \\
\hline Mean AST (IU/L) & $75.71 \pm 44.56$ & $56.84 \pm 25.11^{\star}$ & $42.56 \pm 18.56^{\star}$ \\
Mean ALT (IU/L) & $76.85 \pm 38.37$ & $58.12 \pm 30.82^{\star}$ & $42.99 \pm 22.47^{\star}$
\end{tabular}

* Significantly $(\mathrm{p}<0.05)$ reducued from baseline.

Global assessment of Treatment for Effectiveness and Safety by Investigator

The global assessment of treatment for safety and effectiveness by investigator was assessed in 974 evaluable patients from total included patients. Global effectiveness as graded by investigator was rated as "Excellent", "Good" or "Poor" considering the overall response/improvement seen clinically, biochemically and on ultrasonography (radiologically). The data of global effectiveness showed that a total of 556 (57.08\%) patients had an "Excellent" improvement, 415 (42.61\%) had a "Good" improvement and $3(0.31 \%)$ patient showed a "Poor" improvement (Figure 3).

Similarly, in global safety assessment the investigator rated the overall tolerability of Ensules" soft gelatin capsules as "Excellent", "Good" or "Poor". The data from global safety assessment showed an "Excellent" in 565 (58.01\%) patients and a "Good" in 409 (41.99\%) patients. No investigator had reported "Poor" safety of Vitamin E (400 IU) and F. excelsior (500 mg) soft gelatin capsules. 


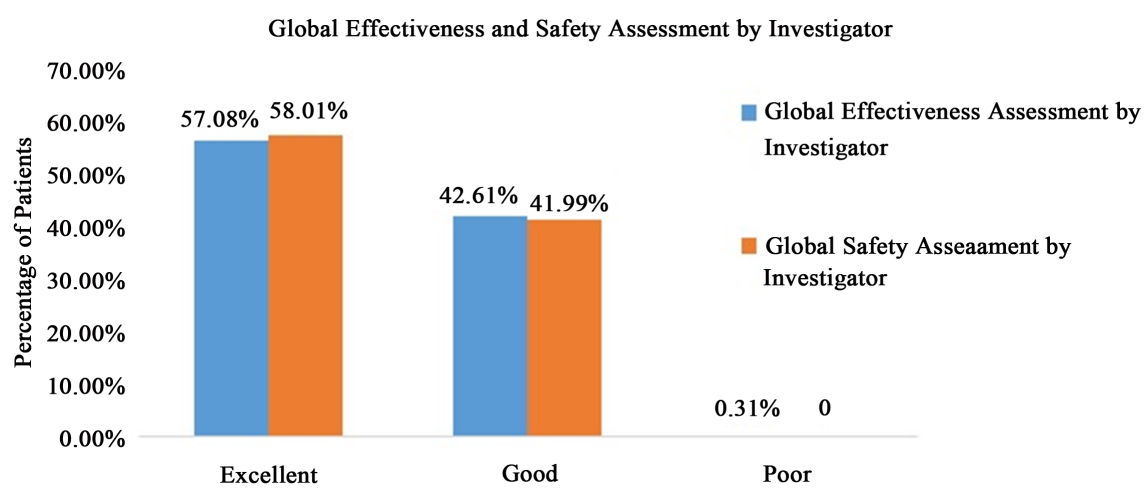

Figure 3. Global effectiveness and safety assessment by investigator.

\section{Discussion}

Nonalcoholic fatty liver disease (NAFLD), is widely prevalent and one of the major cause of chronic liver disease worldwide [1] [2]. There is no definite treatment for NAFLD. Vitamin E an antioxidant is recommended for the management of NAFLD.

A total of 1114 patients were included in our study. At baseline, majority of the patients (71.18\%) had Grade II liver steatosis followed by $21.01 \%$ and $7.81 \%$ patients who had Grade III and Grade I liver steatosis, respectively. After 12 weeks of treatment with vitamin E and Fraxinus excelsior soft gelatin capsules $\left(\right.$ Ensules $\left.^{+}\right), 21 \%$ patients had no steatosis, $58.79 \%$ patients were in Grade 1 steatosis, $19.57 \%$ in grade II steatosis and only $0.63 \%$ patients in Grade III steatosis. The mean percentage reduction in aspartate aminotransferase (AST) level at week 6 and week 12 from baseline was $24.92 \%$ and $43.78 \%$, respectively. Similarly, the mean percentage reduction in alanine aminotransferase (ALT) level at week 6 and week 12 from baseline was $24.37 \%$ and $44.05 \%$ respectively. The mean reductions in AST and ALT were significant at week 6 and week 12. More than $50 \%$ of the investigators rated treatment as excellent for the safety and effectiveness.

Patil et al., in 2018 reported that combination of Vitamin E (400 IU) and F. excelsior $(500 \mathrm{mg}$ ) were found to be more effective than vitamin E monotherapy in the treatment of NAFLD patients by improving the lipid profile and liver function. The study reported significant improvement in liver function parameters with vitamin $\mathrm{E}$ and F. excelsior combination [AST (53.64\%) and ALT (59.53\%) compared to vitamin E 400 IU capsule (AST: $28.45 \%$, ALT: $37.33 \%$ ) twice daily for 8 weeks treatment. It was also observed in this study that combination of Vitamin E (400 IU) and F excelsior $(500 \mathrm{mg}$ ) was found to be significantly effective in improving the liver function parameters (AST, ALT, Alkaline phosphatase (ALP) and Bilirubin) than Vitamin E monotherapy in patients with NAFLD [6]. A randomized three arm controlled trial (PIVENS) conducted by Sanyal AJ et al., on 247 patients who received vitamin E 800 IU once daily for the period of 96 weeks in one arm showed reduction in AST and ALT levels by $21.3 \mathrm{IU} / \mathrm{L}$ and 37 IU/L respectively [5]. There are pre-clinical evidences showing positive effects of F. Excelsior on glucose homeostasis and insulin resistance. Vitamin $\mathrm{E}$ is an 
excellent anti-oxidant and help in neutralizing reactive oxygen species generated due to disturbed metabolism [10] [11]. A meta-analysis of randomized controlled trials conducted by Sato K, et al; found vitamin E significantly improved liver function and histologic changes in patients with NAFLD/NASH. Vitamin E treatment in NAFLD adult patients showed significant reductions in AST of 13.91 IU/L, ALT by $22.44 \mathrm{IU} / \mathrm{L}$, ALP by $10.39 \mathrm{IU} / \mathrm{L}$ and steatosis by $0.67 \mathrm{IU} / \mathrm{L}$ as compared to the control treatment [12].

To the best of our knowledge this is one of the largest data of NAFLD reported from India. The strength of our study is large number of Indian NAFLD patients and real world clinical practice settings. Like any real world study the limitations of our study are fundamentally associated with the design of any real world study with no strict patient selection criterion, comorbidities, variable adherence to treatment, other co-prescribed therapies, etc.

\section{Conclusion}

Evidence from this Indian real-life study suggests that Vitamin E (400 IU) and $F$. excelsior $(500 \mathrm{mg})$ is safe and effective in the treatment of NAFLD in routine clinical practice. Its consumption is associated with improvement in liver steatosis and liver function parameters (AST and ALT). Given the limited therapeutic options in NAFLD, this combination has the potential to bridge the therapeutic gap in management of NAFLD.

\section{Acknowledgements}

We thank all investigators, patients and care givers who participated in this study. We would also like to thank Dr. Sanket Newale, Dr. Rahul Kotwal and Dr. Parthasarathy $\mathrm{M}$. for assistance in drafting and editing the manuscript.

\section{Conflicts of Interest}

Dr. Ashish Mungantiwar, Priyanka Srivastava, Nirali Bhatt, Dr. Dhavalkumar Patel, Dr. Soumen Roy and Dr. Amit Qamra are salaried employees of Macleods Pharmaceuticals Ltd., Mumbai, India. Other authors declare no conflict of interest.

\section{References}

[1] Patel, S.S. and Siddiqui, M.S. (2019) Current and Emerging Therapies for Nonalcoholic Fatty Liver Disease. Drugs, 79, 75-84.

https://doi.org/10.1007/s40265-018-1040-1

[2] Ashtari, S., Purhoseingholi, M.A. and Zali, M.R. (2015) Non-Alcohol Fatty Liver Disease in Asia: Prevention and Planning. World Journal of Hepatology, 7, 1788-1796. https://doi.org/10.4254/wjh.v7.i13.1788

[3] Kalra, S., Vithalani, M., Gulati, G., Kulkarni, C.M., Kadam, Y., Pallivathukkal, J., et al. (2013) Study of Prevalence of Nonalcoholic Fatty Liver Disease (NAFLD) in Type 2 Diabetes Patients in INDIA (SPRINT). Journal of the Association of Physicians of India, 61, 448-453. 
[4] Patel, R., Dosi, R., Joshi, H., Sheth, S., Shah, P. and Jasdanwala, S. (2014) Non-Alcoholic Fatty Liver Disease (NAFLD) in Obesity. Journal of Clinical and Diagnostic Research, 8, 62-66.

[5] Sanyal, A.J., Chalasani, N., Kowdley, K.V., McCullough, A., Diehl, A.M., Bass, N.M., et al. (2010) Pioglitazone, Vitamin E, or Placebo for Nonalcoholic Steatohepatitis. The New England Journal of Medicine, 362, 1675-1685. https://doi.org/10.1056/NEJMoa0907929

[6] Chetan, P., Ashish, M. and Priyanka, S. (2018) Fraxinus excelsior and Vitamin E Combination for NAFLD (FEVEN). The Indian Practitioner Journal, 71, 22-29.

[7] Pacana, T. and Sanyal, A.J. (2012) Vitamin E and Non-Alcoholic Fatty Liver Disease, Current Opinion in Clinical Nutrition and Metabolic Care, 15, 641-648. https://doi.org/10.1097/MCO.0b013e328357f747

[8] Dowman, J.K., Tomlinson, J.W. and Newsome, P.N. (2010) Pathogenesis of Non-Alcoholic Fatty Liver Disease. QJM: An International Journal of Medicine, 103, 71-83. https://doi.org/10.1093/qjmed/hcp158

[9] Saturni, S., Bellini, F., Braido, F., Paggiaro, P., Sanduzzi, A., Scichilone, N., et al. (2014) Randomized Controlled Trials and Real Life Studies Approaches and Methodologies: A Clinical Point of View. Pulmonary Pharmacology \& Therapeutics, 27, 129-138. https://doi.org/10.1016/j.pupt.2014.01.005

[10] Patton, H.M., Yates, K., Unalp-Arida, A., et al. (2010) Association between Metabolic Syndrome and Liver Histology among Children with Nonalcoholic Fatty Liver Disease. The American Journal of Gastroenterology, 105, 2093-2102. https://doi.org/10.1038/ajg.2010.152

[11] Hadi, H., Vettor, R. and Rossato, M. (2018) Vitamin E as a Treatment for Nonalcoholic Fatty Liver Disease: Reality or Myth. Antioxidants, 7, 12. https://doi.org/10.3390/antiox7010012

[12] Sato, K., Gosho, M., Yamamoto, T., Kobayashi, Y., Ishii, N., Ohashi, T., et al. (2015) Vitamin E Has a Beneficial Effect on Nonalcoholic Fatty Liver Disease: A Meta-Analysis of Randomized Controlled Trials. Nutrition, 31, 923-930.

https://doi.org/10.1016/j.nut.2014.11.018 